\title{
Monte Carlo simulation of reverse emittance exchange in MICE
}

\author{
Craig Brown ${ }^{a, b, *}$ on behalf of the MICE collaboration \\ ${ }^{a}$ Brunel University London, \\ Uxbridge, $U K$ \\ ${ }^{b}$ STFC Rutherford Appleton Laboratory, \\ Harwell Oxford, Didcot, UK \\ E-mail: craig.brown@brunel.ac.uk
}

High brightness muon beams for a muon collider can be made from the bombardment of protons against a target producing pions, which subsequently decay into muons. Such a muon beam occupies a large phase-space volume and must be cooled to achieve luminosities suitable for a muon collider. The Muon Ionization Cooling Experiment (MICE) has demonstrated transverse ionization cooling. A muon collider requires both longitudinal and transverse cooling. This can be achieved through a wedge-shaped absorber, where both the longitudinal and transverse phase spaces are simultaneously manipulated during the ionization cooling process. The change in longitudinal and transverse phase space densities obtained from placing a polyethylene wedge into the MICE cooling channel are presented here.

40th International Conference on High Energy physics - ICHEP2020

July 28 - August 6, 2020

Prague, Czech Republic (virtual meeting)

\footnotetext{
${ }^{1}$ The work described here was made possible by grants from Department of Energy and National Science Foundation (USA), the Instituto Nazionale di Fisica Nucleare (Italy), The Science and Technology Facilities Council (UK), the European Community under the European Commission Framework Programme 7 (AIDA project, grant agreement no. 262025, TIARA project, grant agreement no. 261905 and EuCARD), the Japan Society for Promotion of Science and the Swiss National Science Foundation, in the framework of the SCOPES programme. We gratefully acknowledge all sources of support. We are grateful to the staff of ISIS for reliable operation of ISIS. We acknowledge the use of Grid computing resources deployed and operated by GridPP in the UK, http://www.gridpp.ac.uk/.

${ }^{*}$ Speaker
} 


\section{Muon Ionization Cooling Experiment}

The MICE experiment was performed at the Rutherford Appleton Laboratory, Harwell, UK [1]. To generate a muon beam a titanium target was dipped into the ISIS circulating proton beam creating pions, which were subsequently captured in a quadrupole magnet. These pions then decayed to muons in the transport line. Each muon's position and momentum were measured using two tracking detectors immersed in a near uniform multi-Tesla magnetic field either side of an energy-absorbing material. Each tracker consisted of 5 stations of three planes of scintillating fibres. The three planes were at 60 degrees to each other. The muon beam was assembled from each muon's individual position and momentum measurement.

\section{Reverse Emittance Exchange}

When muons are produced by proton collision with a target, the beam has a large emittance and occupies a large phase-space volume in position-momentum space. To meet the acceptance requirements of a storage ring, these muons must be cooled, with ionization cooling the only viable process to cool the muon beam to the required phase-space density on a shorter timescale than the muon lifetime. Ionization cooling only reduces the transverse emittance. Emittance Exchange allows the transfer of emittance between longitudinal and transverse phase-space, and thus overall $6 \mathrm{D}$ cooling can be achieved.

In Reverse Emittance Exchange the beam is first passed through a wedge of specific thickness creating a momentum spread and a position-energy correlation. The beam is then passed through a magnetic dipole reducing the beams's transverse position spread. This allows one to increase the transverse phase-space density at the expense of decreased longitudinal phase-space density.
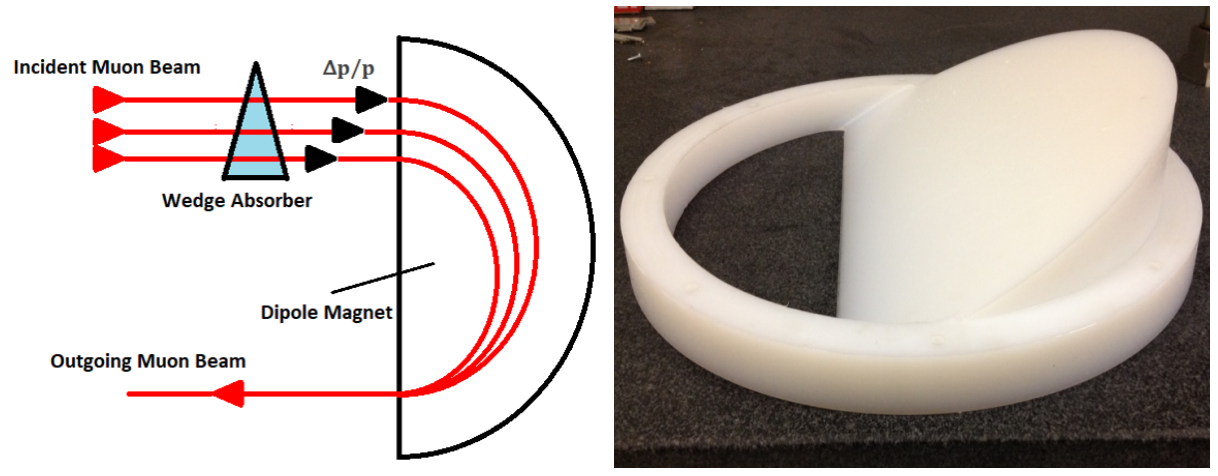

Figure 1: Left: Reverse Emittance Exchange Principle. Right: Polyethylene Wedge used in MICE

\section{Monte Carlo Simulation of the Change in Phase-Space Density}

The phase-space densities upstream and downstream of when no wedge and a wedge are inserted into the MICE beamline are shown in Figure 2, the input beam having a nominal transverse emittance of $6 \mathrm{~mm}$ and $140 \mathrm{MeV} / \mathrm{c}$ momentum [2]. Two significant sources of bias on the calculated 
phase-space density (due to sample selection) have not yet been accounted for. First, a transmission cut bias, which excludes particles which have escaped beyond the aperture of the experiment before a downstream Time-of-Flight detector at the end of the cooling channel. Those particles have likely heated. Secondly, the wedge creates a momentum dispersion in the beam. The momentum and position measurements of the beam take place at fixed longitudinal planes. The differing arrival times of muons with differing longitudinal momentum creates an apparent change in the transverse phase-space density at the downstream measurement planes and needs to be corrected for.

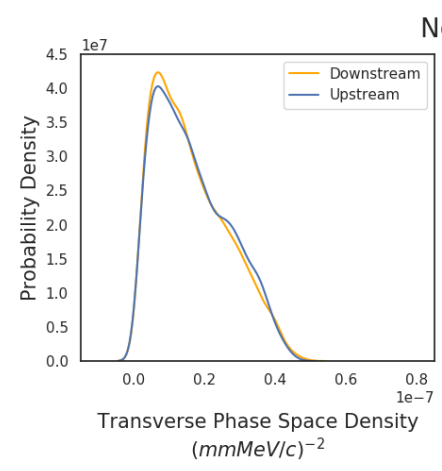

No Absorber MC Truth Phase Space Densities
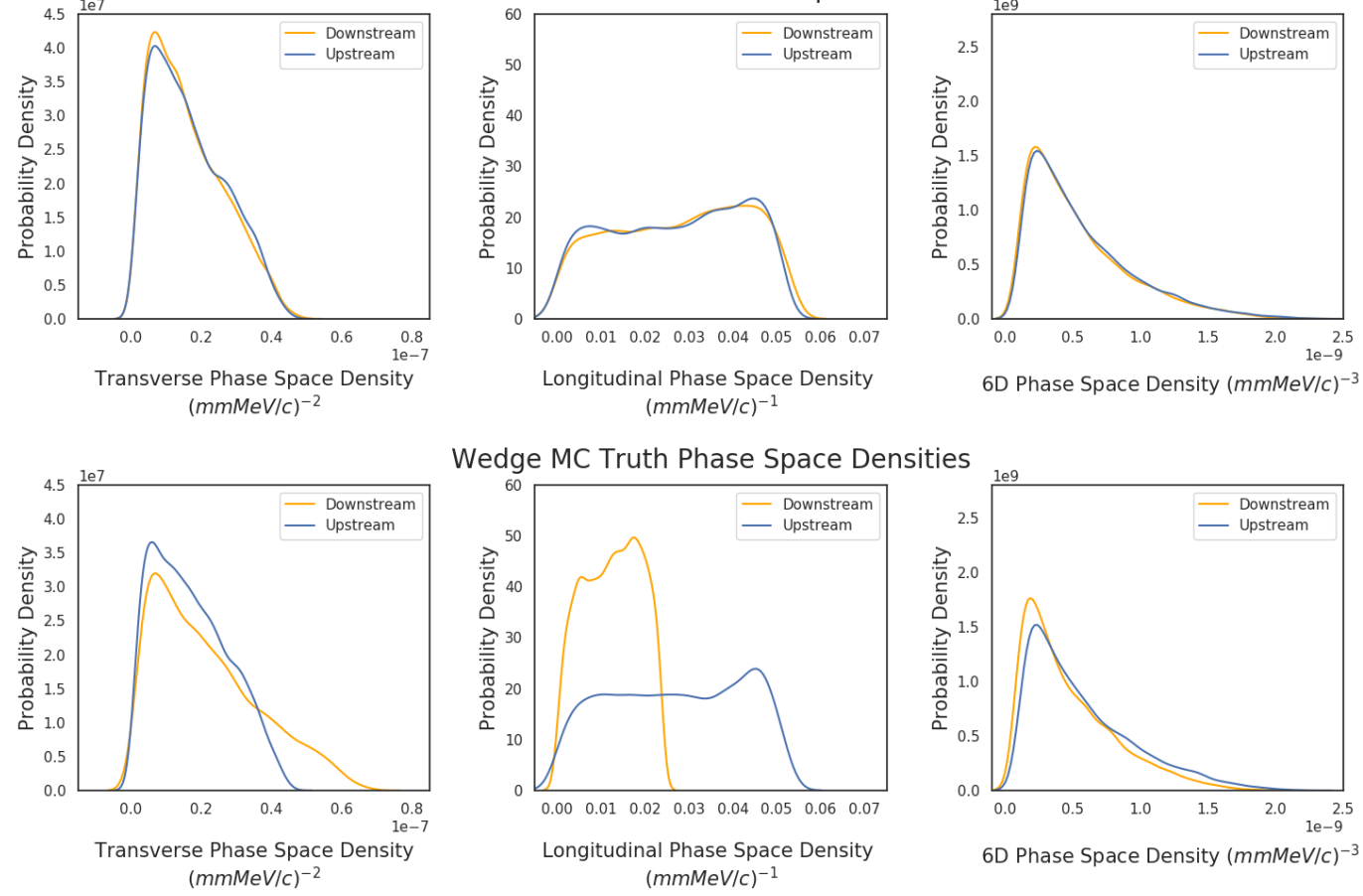

Wedge MC Truth Phase Space Densities
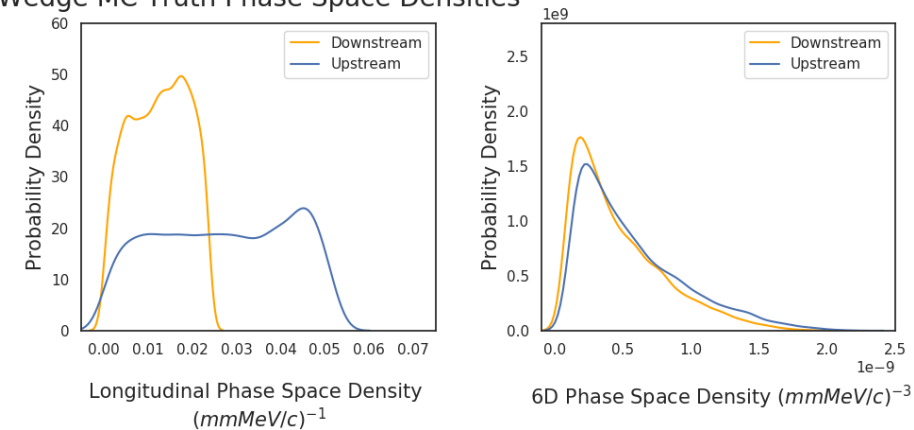

Figure 2: MC simulation of the Upstream and Downstream Transverse (left), Longitudinal (middle) and 6D (right) phase-space densities when no wedge (top) and a wedge (bottom) is present in the MICE beamline.

\section{Conclusion}

The conservation of the $6 \mathrm{D}$ phase space density when no wedge is present in the MICE beamline shows the potential of this analysis to get an unbiased result not affected by magnet optic effects. The Emittance Exchange effect can then be quantified once biases have been corrected for.

\section{References}

[1] MICE collaboration., Bogomilov, M., Tsenov, R. et al. Demonstration of cooling by the Muon Ionization Cooling Experiment, Nature (578) 53-59.

[2] Brown, C. (2020). Monte Carlo Simulation of Reverse Emittance Exchange in MICE, ICHEP 2020, Prague, Czech Republic, Poster 55. 